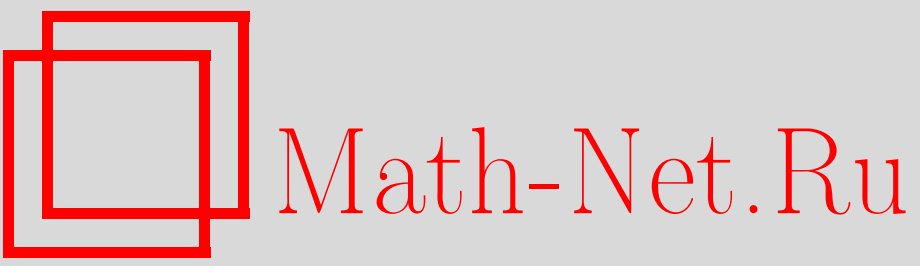

А. В. Гришин, Многообразие ассоциативных колец не шпехтово, УМН, 1999, том 54, выпуск 5, 157-158

DOI: https://doi.org/10.4213/rm209

Использование Общероссийского математического портала Math-Net.Ru подразумевает, что вы прочитали и согласны с пользовательским соглашением

http://www.mathnet.ru/rus/agreement

Параметры загрузки:

IP : 54.89 .56 .158

26 апреля 2023 г., 15:51:49 


\title{
МНОГООБРАЗИЕ АССОЦИАТИВНЫХ КОЛЕЦ НЕ ШПЕХТОВО
}

\author{
А.В. Гришин
}

С конца 60-х годов и до недавнего времени в группах, алгебрах Ли и некоторых кольцах, близких к ассоциативным, были построены примеры объектов, не имеющих конечного базиса тождеств. С другой стороны, для ассоциативного случая (а также для некоторых других) был получен ряд положительных результатов весьма общего характера. Для конечных колец - теорема Львова-Крузе [1], [2] (см. также [3]), для алгебр над полем характеристики 0 - теорема Кемера [4], для $T$-пространств (некоторое обобщение $T$-идеалов) над полем нулевой характеристики, а также в частных случаях над полем характеристики $p>0$ - результаты автора [5], [6]. На некотором этапе у ряда специалистов в $P I$-теории стало складываться впечатление, что в ассоциативном случае любая алгебра над достаточно "хорошим" кольцом (например, над полем или над кольцом целых чисел) имеет конечный базис тождеств. Однако это не так.

Прежде чем формулировать основной результат, приведем следующее

ОПРЕДЕлЕниЕ. Пусть $k$ - ассоциативное и коммутативное кольцо с единицей, $F=k\left\langle x_{1}, \ldots\right.$, $\left.x_{i}, \ldots\right\rangle$ - свободная счетнопорожденная ассоциативная $k$-алгебра. Назовем $T$-модулем алгеб$p$ bl $F$ всякий ее $k$-подмодуль $V$, замкнутый относительно подстановок вместо переменных $x_{i}$ произвольных элементов из алгебры $F$, в частности, если $k$ - поле, то $V-T$-nространство, если $k=\mathbb{Z}$-кольцо целых чисел, то $V-T$-группа. Если $T$-модуль $V$ является в то же время и идеалом, то он называется $T$-идеалом.

Цель настоящей работы - показать, что в свободной $\mathbb{Z}$-алгебре $\mathbb{Z}\left\langle x_{1}, \ldots, x_{i}, \ldots\right\rangle$ существуют бесконечные возрастающие цепочки $T$-идеалов. Кроме того, будет показано, что в относительно свободной алгебре $\mathbb{Z}\left\langle x_{1}, \ldots, x_{i}, \ldots\right\rangle /\left(\left[x_{1},\left[x_{2}, x_{3}\right]\right]\right)^{T}$ существуют бесконечные возрастающие цепочки $T$-подгрупп. Будут даны явные конструкции. Имеет место следующая

Теорема 1. Пусть $k$ - поле характеристики $2, F=k\left\langle x_{1}, \ldots, x_{i}, \ldots\right\rangle$ - свободная счетнопорожденная ассочиативная $k$-алгебра, $\Omega-T$-идеал алгебры $F$, порожденньи многочленом $\left[x_{1},\left[x_{2}, x_{3}\right]\right]$. Тогда система $S$, состоящая из многочленов $f_{n}=x_{1}^{2} \cdots x_{n}^{2}$, $n \in \mathbb{N}$, порождает в относительно свободной алгебре $F / \Omega$ не конечно базируемое T-пространство $S^{T}$.

НАБРОсок докАЗАтЕЛЬСтвА. (1) Рассмотрим сначала $T$-пространство $S^{T}+\Omega$ в свободной алгебре $F$. Достаточно показать, что для любого $n>1$ многочлен $f_{n}$ не лежит в $T$-пространстве $\left\{f_{1}, \ldots, f_{n-1}\right\}^{T}+\Omega$. Допустим противное. Легко видеть, что тогда можно считать, что многочлен $f_{n}$ является суммой некоторого элемента из $\Omega$ и многочленов вида $g=h_{1} \cdots h_{r}$, где $1 \leqslant r \leqslant n-1$, многочлен $h_{i}$ либо равен квадрату полилинейного одночлена, либо имеет вид $u v+v u$, причем $\operatorname{deg}_{x_{i}} g=2$ при $i=1, \ldots, n$.

Скажем, что $g$ - квадратичный одночлен, если все $h_{i}$ - квадраты полилинейных одночленов, причем хотя бы один из этих одночленов имеет степень $\geqslant 2$.

Скажем, что $g$ - коммутаторный многочлен, если среди сомножителей $h_{i}$ присутствуют многочлены вида $u v+v u$.

Предположение о том, что одночлен $f_{n}$ принадлежит $T$-пространству $\left\{f_{1}, \ldots, f_{n-1}\right\}^{T}+\Omega$, равносильно тому, что $f_{n}$ является суммой квадратичных одночленов, коммутаторных многочленов и элемента из $\Omega$.

Для того чтобы привести это к противоречию, нам понадобится следующая вспомогательная коммутативная алгебра $A=k\left[\left\{a_{i j} \mid i, j \in \mathbb{N}\right\}\right]$, где коммутирующие между собой элементы $a_{i j}$ удовлетворяют следующим соотношениям: $\alpha_{i j}=\alpha_{j i}, \alpha_{i j}^{2}=1,\left(1+\alpha_{i j}\right)\left(1+\alpha_{i l}\right)=0$, $\left(1+\alpha_{i j}\right)\left(1+\alpha_{k l}\right)=\left(1+\alpha_{i k}\right)\left(1+\alpha_{j l}\right)$, где $i, j, k, l$-попарно различные индексы.

(2) Рассмотрим теперь свободную счетнопорожденную алгебру $\Phi=A\left\langle x_{1}, \ldots, x_{i}, \ldots\right\rangle$, содержащую $k$-подалгебру $F$, в ней идеал $I$, порожденный элементами вида $x_{i} x_{j}+\alpha_{i j} x_{j} x_{i}$, и фактор-алгебру $\Phi_{2}=\Phi / I$. 
Вычисления в алгебре $\Phi_{2}$ показывают, что

а) в алгебре $\Phi_{2}$ выполнено тождество $\left[x_{1},\left[x_{2}, x_{3}\right]\right]=0$;

б) любой коммутаторный многочлен "исчезает" в алгебре $\Phi_{2}$;

в) образ одночлена $f_{n}$ в алгебре $\Phi_{2}$ не может быть представлен в виде суммы образов квадратичных одночленов.

Это и приводит к противоречию.

Следующее утверждение является некоторым техническим следствием (правда, нетривиальным) теоремы 1.

Теорема 2. Пусть $k$ - поле характеристики 2. Тогда T-идеал, порожденньй в свободной алгебре $k\left\langle x_{1}, \ldots, x_{i}, \ldots, y_{1}, y_{2}, z_{1}, z_{2}\right\rangle$ мноосеством многочленов

$$
g_{0}=y_{1}^{4} z_{1}^{4} z_{2}^{4} y_{2}^{4} y_{1}^{4} z_{1}^{4} z_{2}^{4} y_{2}^{4}, \quad g_{n}=y_{1}^{4} z_{1}^{4} x_{1}^{2} \cdots x_{n}^{2} z_{2}^{4} y_{2}^{4} y_{1}^{4} z_{1}^{4} x_{n+1}^{2} \cdots x_{2 n}^{2} z_{2}^{4} y_{2}^{4},
$$

где $n \in \mathbb{N}$, не является конечно порожденным.

Следствия. 1. Пусть $A$ - коммутативное кольщо, имеющее сюрвективный гомоморфизм на $\mathbb{Z}_{2}$-алгебру с единицей (например, $A=\mathbb{Z}$ ). Тогда указанные в теоремах 1 и 2 многочлены порождают в алгебре $A\left\langle x_{1}, \ldots, x_{i}, \ldots, y_{1}, y_{2}, z_{1}, z_{2}\right\rangle$ не конечнопорохсденный $T$-идеал (T-модуль, T-группу).

2. Многообразие ассоциативных колец не шпехтово. Более того, многообразие ассоциативных ниль-колец индекса 32 не шпехтово.

\section{СПИСОК ЛИТЕРАТУРЫ}

[1] Львов И.В. // Алгебра и логика. 1973. Т. 12. С. 269-297. [2] Kruse В. I. // J. Algebra. 1973. V. 26. P. 298-318. [3] Латышев В. Н. // УМН. 1977. Т. 32. № 4. С. 259-260. [4] Кемер А. Р. // Алгебра и логика. 1987. № 5. С. 597-641. [5] Гришин А. В. // Фундамент. и прикл. матем. 1995. Т. 1. № 3. С. 669-700. [6] Grishin A. V. // Proc. of the First International Moscow-Tainan Alg. Workshop (Tainan, 1994) / ed. Y. Fong et al. Berlin: de Gruyter, 1996. P. 225-227.

Московский педагогический

Принято редколлегией государственный университет 23.08.1999 